\title{
Environnement urbain
}

Urban Environment

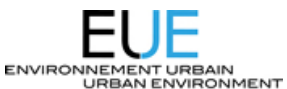

\section{Sfax, laboratoire du développement urbain durable en Tunisie?}

\section{Ali Bennasr, Taoufik Megdiche et Eric Verdeil}

\section{Volume 7, 2013}

Villes arabes, villes durables? Enjeux, circulations et mise à l'épreuve de nouvelles politiques urbaines

Arab cities, sustainable cities? Challenges, movements and testing of new urban policies south of the mediterranean

URI : https://id.erudit.org/iderudit/1027728ar

DOI : https://doi.org/10.7202/1027728ar

Aller au sommaire du numéro

Éditeur(s)

Réseau Villes Régions Monde

ISSN

1916-4645 (numérique)

Découvrir la revue

Citer cet article

Bennasr, A., Megdiche, T. \& Verdeil, E. (2013). Sfax, laboratoire du développement urbain durable en Tunisie? Environnement urbain / Urban

Environment, 7, a83-a98. https://doi.org/10.7202/1027728ar

\section{Résumé de l'article}

Les initiatives réalisées au nom du développement durable sont souvent critiquées pour leur prise en compte insuffisante des avis et des enjeux locaux, et accusées de se contenter de reproduire des bonnes pratiques décontextualisées. C'est particulièrement le cas dans les pays arabes à régime autoritaire. Cet article revient sur l'expérience de la Stratégie de développement du Grand Sfax (SDGS), considérée comme un succès par plusieurs acteurs internationaux, tunisiens et sfaxiens, afin d'identifier les facteurs de cette réussite relative, tout en soulignant les limites de cette expérience. L’article présente d'abord les débats sur le développement urbain durable dans les pays arabes et notamment dans le contexte tunisien. Puis il analyse l'expérience de Sfax en soulignant le rôle initiateur des institutions internationales, la nature des interactions entre les participants à l'élaboration de cette stratégie et en particulier le rôle des acteurs locaux issus de la société civile, et l'argumentation utilisée qui a permis l'établissement d'un consensus. À travers deux projets issus de cette stratégie, l'article considère l'inscription dans l'histoire locale du développement comme une condition essentielle de la poursuite de ce processus. Toutefois, en s'attachant aux évolutions de ces projets jusqu'à la période actuelle marquée par le changement politique, l'article souligne aussi les limites du consensus atteint, et notamment les tensions qui surviennent autour de l'enjeu social.
Ce document est protégé par la loi sur le droit d'auteur. L'utilisation des services d'Érudit (y compris la reproduction) est assujettie à sa politique d'utilisation que vous pouvez consulter en ligne.

https://apropos.erudit.org/fr/usagers/politique-dutilisation/ 


\title{
SFAX, LABORATOIRE DU DEVELOPPEMENT URBAIN DURABLE EN TUNISIE?
}

\author{
Ali BENNASR' \\ Taoufik MEGDICHE \\ Eric VERDEIL ${ }^{3}$
}

\begin{abstract}
† RÉSUMÉ
Les initiatives réalisées au nom du développement durable sont souvent critiquées pour leur prise en compte insuffisante des avis et des enjeux locaux, et accusées de se contenter de reproduire des bonnes pratiques décontextualisées. C'est particulièrement le cas dans les pays arabes à régime autoritaire. Cet article revient sur l'expérience de la Stratégie de développement du Grand Sfax (SDGS), considérée comme un succès par plusieurs acteurs internationaux, tunisiens et sfaxiens, afin d'identifier les facteurs de cette réussite relative, tout en soulignant les limites de cette expérience. L'article présente d'abord les débats sur le développement urbain durable dans les pays arabes et notamment dans le contexte tunisien. Puis il analyse l'expérience de Sfax en soulignant le rôle initiateur des institutions internationales, la nature des interactions entre les participants à l'élaboration de cette stratégie et en particulier le rôle des acteurs locaux issus de la société civile, et l'argumentation utilisée qui a permis l'établissement d'un consensus. À travers deux projets issus de cette stratégie, l'article considère l'inscription dans l'histoire locale du développement comme une condition essentielle de la poursuite de ce processus. Toutefois, en s'attachant aux évolutions de ces projets jusqu'à la période actuelle marquée par le changement politique, l'article souligne aussi les limites du consensus atteint, et notamment les tensions qui surviennent autour de l'enjeu social.
\end{abstract}

MOTS-CLÉS - Urbanisme, développement durable, environnement, concertation, participation, société civile, régime autoritaire, Sfax, Tunisie

\begin{abstract}
I ABSTRACT
Initiatives on behalf of sustainable development are often criticized for inadequate consideration and advice of local issues and replication of decontextualized good practices only. This is particularly the case in Arab countries with authoritarian regimes. This article reviews the experience of the Development Strategy of Greater Sfax, considered as a success by many international, Tunisian and Sfaxian actors. We seek to identify the factors behind this relative success, while emphasizing the limits of this experiment. The article first presents the debate on sustainable urban development in Arab countries and especially in the Tunisian context. Then it analyzes the experience of Sfax emphasizing the role of international institutions which played a major role in launching the process, the nature of interactions between participants in the strategy development, including the role taken by local actors from the civil society, and the argumentation used to reach this consensus. Examining two projects from this strategy, the paper considers the inclusion in the local history of development as an essential condition for the continuation of this process. However, by focusing on the development of these projects until the current period of political change, the article also highlights the limits of consensus, including in particular the tensions that arise around the social issue.
\end{abstract}

KEYWORDS - Urban planning, sustainable development, environment, consultation, participation, civil society, authoritarian regime, Sfax, Tunisia

\footnotetext{
' Ali Bennasr, professeur de Géographie à l'université de Sfax, Directeur du laboratoire de recherches SYFACTE. Ses recherches comme ses encadrements de thèses portent sur la ville et son aménagement. II a participé à plusieurs actions de recherches dont la stratégie de développement du grand Sfax (SDGS 2016). II coordonne des projets de recherches dans le cadre de coopération scientifique entre la Tunisie et la France.

${ }^{2}$ Taoufik Megdiche est enseignant chercheur à l'Université de Sfax. Domaines de recherche: aménagement, géographie sociale.

${ }^{3}$ Éric Verdeil est chargé de recherche CNRS à l'Université de Lyon, laboratoire Environnement Ville Société (UMR 5600). Ses travaux portent sur les réformes des services urbains, sur les enjeux territoriaux et les renouvellements de l'action publique, à travers l'exemple des réseaux d'eau et d'électricité. Sur divers terrains situés au sud et à l'est de la Méditerranée.
}

I Coordonnées des auteurs : Ali Bennasr [abennasr@hotmail.fr), Université de Sfax, Taoufik Megdiche, Université de Sfax, (megdichetaoufik@yahoo.fr) Eric Verdeil, Université de Lyon-CNRS (eric.verdeil@normalesup.org) 


\section{INTRODUCTION : LES CRITIQUES DU DEVELOPPEMENT URBAIN DURABLE ET LA PRISE EN COMPTE DE LA DIMENSION LOCALE}

Dans les contextes sud-méditerranéens, marqués par des régimes autoritaires, les politiques conduites au nom du développement durable résultent le plus souvent d'une combinaison entre une injonction émanant des institutions extérieures au pays et une approche dirigiste de la part des États, qui contrôlent et transposent cette injonction dans leur législation et leurs institutions (Barthel et al., 2013). De ce fait, les politiques de développement urbain durable font l'objet de diverses critiques: instrumentalisation au bénéfice des gouvernants, mise en avant des solutions techno-environnementales faisant la part belle à des entreprises privées, déficit de participation des acteurs locaux, notamment des collectivités territoriales et sociétés civiles, et en conséquence déficit potentiel d'adéquation aux enjeux locaux (voir Myllylä et Kuvaja, 2005).

Plusieurs travaux menés au Maroc soulignent ces limites. L'analyse des dispositifs participatifs mis en œuvre dans le cadre des Agendas 21 fait ainsi apparaître à la fois l'étroitesse des milieux mobilisés et le contrôle serré des enjeux laissés à la discussion (Navez-Bouchanine, 2007). L'exemple de Marrakech aboutit à des résultats similaires, auxquels s'ajoutent d'autres dimensions (Berry-Chikhaoui et HaouesJouve, 20II). Le processus d'élaboration de l'Agenda 21 peut constituer un moment d'accès à des ressources immatérielles pour des acteurs locaux (notamment associatifs), tandis qu'il est vu par les acteurs municipaux, marginalisés par un pilotage ministériel, comme un espace concurrent de leurs pratiques de légitimation locale (par la distribution de subventions aux associations clientes).

Le cas tunisien permet de pointer d'autres limites de ces processus d'élaboration des politiques de développement urbain durable. En effet, l'acteur étatique joue dans ce pays un rôle de chef d'orchestre des politiques menées au nom du développement durable, notamment en milieu urbain, où les institutions locales apparaissent fortement marginalisées dans l'élaboration des décisions et dans leur mise en œuvre. Les travaux qui ont porté sur le Grand Tunis ont particulièrement mis en évidence ces dimensions, que ce soit à propos du réaménagement des lacs et sebkhas entourant la capitale (Barthel, 2006) ou des politiques d'élaboration d'Agendas 21 dans les petites villes proches de la capitale (Kahloun, 2010). L'opacité des modes de décision dans un contexte autoritaire qui privilégie les allégeances partisanes dans le cadre de relations clientélistes est notamment une explication mise en avant pour comprendre ce fait.

Ces modes d'élaboration condamnent-ils complètement l'espoir de construire des politiques de développement urbain durable correspondant à une démarche véritablement ancrée dans le territoire et prenant en compte les aspirations des acteurs locaux ?

II semble prudent de ne pas surestimer l'ampleur de l'imposition du contenu des politiques de développement durable par les acteurs extérieurs. Divers travaux soulignent en effet que même dans un contexte de relations asymétriques sur le plan financier ou intellectuel, la circulation des modèles ne peut se réduire à l'action des acteurs extérieurs (institutions et consultants internationaux). Les acteurs locaux et nationaux jouent un rôle essentiel de sélection, d'adaptation en fonction de leur capacité et de leurs propres agendas (Robinson 201 I; Souami, 20II). Dans cette perspective, un facteur potentiellement très important est à prendre en considération: le partage d'une vision locale de développement peut conduire à une problématisation qui permet, au-delà des inflexions thématiques imposées par la rhétorique aménageuse du moment (ici le développement durable), de réinscrire les revendications dans une histoire locale de l'aménagement. C'est ce que Marcel Roncayolo, dans un contexte très différent, appelle "l'imaginaire d'une ville » (Roncayolo, 1990).

Notre hypothèse dans cet article est double: plutôt que de voir le développement urbain durable uniquement comme un ensemble de politiques de référence mises en circulation à l'échelle globale et nationale par des acteurs dotés de fortes ressources politiques, institutionnelles et financières, dans une logique top-down, nous postulons l'existence de processus de revendication, d'apprentissage et finalement de co-construction de l'action publique environnementale par des acteurs locaux. Ce processus de revendication et d'apprentissage fait une place importante aux dimensions sociales et participatives, davantage présentes que certaines visions critiques de ce type de politiques citées cidessus ne le reconnaissent. Mais les particularités du contexte local sont déterminantes et nourrissent directement le contenu de l'action publique. Nous proposons ainsi l'idée que les politiques environnementales urbaines font l'objet de négociations et de transformations sur le moyen terme ( 10 à 15 ans), et sont de ce fait également infléchies par les reformulations successives des «modèles» du développement durable, les évolutions 
des enjeux socio-économiques ainsi que les grandes transitions politiques. Pour défendre ces hypothèses, nous retenons une grille de lecture attentive à deux thématiques.

En premier lieu, il s'agit de mesurer l'ouverture du processus d'élaboration de ces politiques dites du développement urbain durable, et notamment le rôle que vont y jouer les représentants des collectivités locales, les municipalités, et ceux de divers groupes locaux, comme les associations, les universitaires, etc. L'objectif ici est de caractériser la manière dont ces derniers parviendront à se faire une place dans le processus d'élaboration des politiques et à infléchir les décisions. Cet examen implique de comprendre les ressources de ces différents acteurs, en termes d'appui externes (par exemple, les opportunités offertes par la coopération internationale) ou internes (jeu de réseau à l'échelle locale ou au sein des institutions nationales).

En deuxième lieu, il s'agit également d'identifier comment les politiques construites au nom du développement urbain durable se saisissent d'enjeux locaux et comment ces derniers peuvent infléchir les modèles prêts à être appliqués qui sont diffusés par les manuels de bonne pratique. Pour effectuer cette lecture, il faut analyser le discours sur le local et ses problèmes tel qu'il est porté par les participants au processus. Cela suppose notamment de faire un allerretour entre les objectifs progressivement élaborés dans le projet et l'histoire de l'aménagement du lieu concerné, pour repérer les continuités ou discontinuités existantes ou revendiquées.

Ce travail est effectué ici à partir de l'exemple de Sfax, deuxième agglomération de Tunisie, qui est apparue dans les dix à quinze dernières années comme un site privilégié d'expérimentation du développement durable, et cela sans volonté nationale d'en faire un site pilote. Ce sont plutôt l'enchevêtrement d'un contexte local très dégradé sur le plan environnemental, les mobilisations conjuguées d'acteurs locaux bien enracinés, la volonté des bailleurs de fonds de promouvoir de nouvelles logiques d'action qui ont abouti, progressivement, à une maturation spécifique, qui a pris la forme d'une stratégie de développement du Grand Sfax. II ne faut pour autant exagérer ni l'ampleur, ni les résultats de cet exercice. Les changements politiques en cours représentent à cet égard un nouveau défi pour ces acteurs locaux, confrontés à la montée de nouvelles revendications qu'il faut prendre en charge.

Les éléments d'analyse mobilisés pour cette réflexion proviennent, pour une large part, d'une réflexion critique rétrospective sur la participation de deux des auteurs au processus en question, de 2002 jusqu'à aujourd'hui. Par ailleurs, ces réflexions critiques s'ancrent dans des travaux de recherche comparatifs menés dans le cadre du projet AUF-Mersi «Bilan des premières expérimentations de la ville durable au Sud de la Méditerranée » et de deux autres recherches (Services urbains en réseau dans les villes moyennes du sud de la Méditerranée, financement AUF, 2007-2009 et Villes et efficience énergétique en Tunisie - programme Utique 2009).

\section{LE DÉVELOPPEMENT URBAIN DURABLE EN TUNISIE : INSTITUTIONS ET PRATIQUES}

L'objectif de cette première section est, dans un premier temps, de présenter dans ses grandes lignes le dispositif institutionnel mis en place par le gouvernement tunisien pour promouvoir des politiques de développement durable, notamment dans les villes, et dans un deuxième temps, de faire ressortir les limites de ce dispositif, qui sont examinées à travers les travaux existants.

\section{I.I La Tunisie, un bon élève du développement durable?}

Les autorités tunisiennes ont été très réactives pour adapter leur système institutionnel en réponse aux impulsions données par la communauté internationale en faveur de politiques de protection de l'environnement et du développement durable. La création du programme METAP (Programme d'action pour la Méditerranée lancé par la Banque mondiale ${ }^{4}$ ) et le Sommet mondial de Rio ont joué un rôle majeur et conduit à la création en Tunisie de plusieurs institutions et programmes, comme l'Agence nationale pour la protection de l'environnement (1988), le ministère de l'Environnement et de l'Aménagement du territoire (1991), la Commission nationale du développement durable (1993), etc. Suivent ensuite la promulgation d'un nouveau Code de l'aménagement du territoire et de l'urbanisme intégrant cette problématique (1994), l'Observation nationale du développement durable (1994) et le lancement de l'Agenda 21 national en 1995 (Gafsi, 20I0; 20II). À partir de la fin des années 1990, la thématique du développement durable se décline à l'échelle urbaine. Ainsi, soixante communes tunisiennes se sont engagées dans des Agendas 2I, cinq autres ont adhéré

\footnotetext{
${ }^{4}$ METAP : Mediterranean Technical Assistance Programme, créé en 1990 par la Banque mondiale, la Banque Européenne d'investissement et le Programme des Nations-Unies pour le développement.
} 
au programme Gold de développement de la gouvernance locale (Kairouan, Kef, Kasserine, Mahdia, Zaghouan), et enfin, Tunis et Sfax se sont engagées dans des Stratégies de Développement des Villes (SDVT).

Cette adhésion officielle aux objectifs du développement durable doit s'analyser comme une stratégie du régime, alors engagé dans une politique d'ajustement structurel, pour donner sur la scène internationale des gages au sujet de sa modernité et de sa volonté de réforme. Au niveau interne, cette mobilisation institutionnelle autour du thème de l'environnement et du développement durable correspond également à une mise en scène par le régime de sa propre légitimité. La multiplication des boulevards de l'environnement, dans les années 1990, est emblématique de cet effet d'affichage (voir Photo I).

\section{I.2 Le Développement urbain durable tunisien en pratique}

Un certain nombre de travaux et de témoignages permettent d'analyser le bilan de ces initiatives, en se focalisant ici sur les projets à dimension urbaine. Ces éléments font ressortir des contradictions fortes entre les ambitions affichées de transformation des objectifs vers une prise en compte des dimensions environnementale et sociale et la réalité des pratiques. Celles-ci témoignent à la fois d'une persistance de la priorité donnée au développement économique (et aux intérêts pécuniaires des bénéficiaires directs des opérations) et du caractère souvent mécanique des exercices menés, notamment du point de vue de la participation du public. Les documents réalisés relèvent de pratiques d'expertise classique, tandis que le bénéfice des réalisations est confisqué par les détenteurs de l'autorité politique ou de leur clientèle, dans un contexte de forte opacité des transactions.

Les opérations d'aménagement des lacs et sebkhas de Tunis ont constitué depuis les années 1980, et notamment durant les années 1990 et 2000, des occasions fortes de faire émerger de nouvelles pratiques combinant développement urbain et préservation ou réhabilitation des écosystèmes lacustres (Barthel, 2006; 2008a). Ces projets s'inscrivent d'ailleurs dans de nouvelles relations partenariales avec des bailleurs de fonds étrangers, dont certains explicitement orientés vers des pratiques de gestion durable (projet Cité Durable, financé par le PNUD en 1994, pour la sebkha Sijoumi). Toutefois, les arbitrages rendus ont conduit en fin de compte à la marginalisation de la dimension de préservation dans trois des quatre projets, et ont donné la priorité aux enjeux de développement économique et immobilier. Certaines de ces décisions paraissent marquées par l'affairisme, notamment le

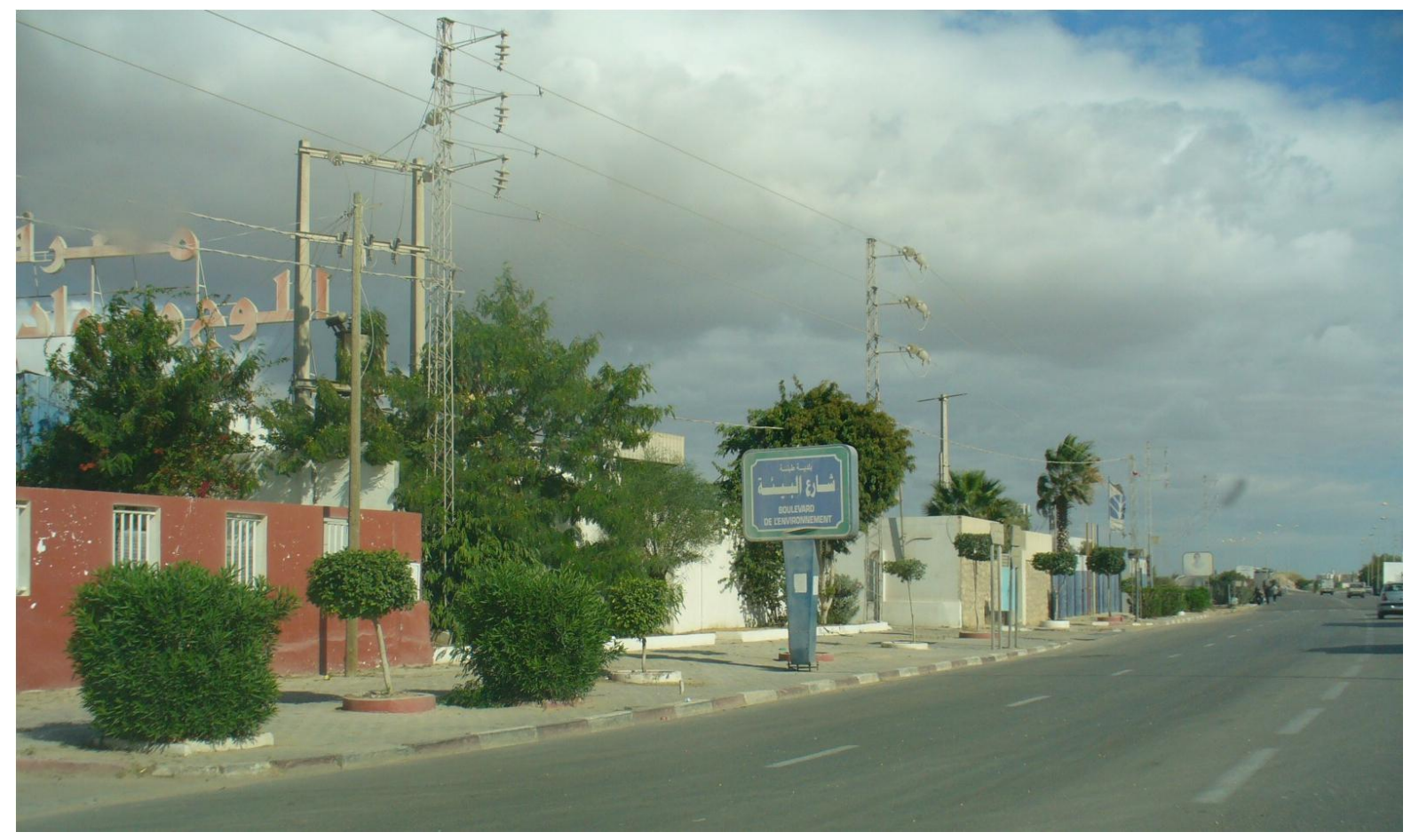

Fig. I - Le Boulevard de l’Environnement à Sfax en 2005 (cliché Eric Verdeil) 
sacrifice des pinèdes riveraines du Lac Nord de Tunis face à des perspectives mirobolantes d'urbanisme inspiré du modèle de Dubaï (Barthel, 2008b; Chabbi, 2012).

L'exemple précédent présente une forte spécificité liée au statut de Tunis comme capitale et à l'importance des enjeux financiers dans les projets d'aménagement en question. C'est pourquoi il est utile d'examiner en contrepoint les résultats disponibles au sujet de l'élaboration et de la mise en œuvre de politiques de développement durable, à travers l'instrument des Agendas 2I, dans des petites villes de la périphérie tunisoise (Kahloun, 20I0). Pour l'auteur du travail cité, l'Agenda $2 \mathrm{I}$ a l'avantage de permettre une prise en compte plus effective des enjeux environnentaux grâce à l'implication, dans le processus d'élaboration, de citoyens qui suppléent le manque de vision et de compétences de l'administration locale. Toutefois, ce document ne permet aucunement de débloquer le contrôle exercé par l'État sur l'urbanisation, qui reste tributaire de canaux de décisions opaques et clientélistes. En fin de compte, les intentions exprimées par l'Agenda 21 restent donc largement lettre morte.

Les mécanismes d'institutionnalisation du développement urbain durable en Tunisie sont divers et témoignent d'une volonté précoce de l'État tunisien de satisfaire les bailleurs de fonds internationaux en adoptant les signes extérieurs des nouvelles orientations. Le développement urbain durable revêt donc dans cette perspective une dimension de légitimation du régime à vocation externe (permettant la poursuite de l'aide internationale) mais également interne (en manifestant aux yeux des citoyens une étape nouvelle sur la voie de la modernisation et du développement). Toutefois, les modes de décision restent essentiellement descendants et opaques. Les nouveaux modes de délibération, recourant à la participation, institués dans le cadre de certains projets estampillés de développement urbain durable, apparaissent comme des alibis, sans être apparemment de nature à modifier les pratiques. C'est ce constat globalement pessimiste que nous examinons plus en détail à travers l'exemple de Sfax.

\section{LA STRATÉGIE DE DÉVELOPPEMENT DU GRAND SFAX : UN PROCESSUS ORIGINAL DE CONSTRUCTION D'UNE POLITIQUE LOCALE DE DÉVELOPPEMENT URBAIN DURABLE}

La Stratégie de développement du Grand Sfax (SDGS) est un processus d'élaboration d'une politique de développement local incluant, comme le font les exercices similaires, une forte dimension de développement durable (Robinson, 20II). Les Stratégies de développement de ville (City Development Strategies ou CDS) sont :

\begin{abstract}
an action-oriented process, developed and sustained through participation, to promote equitable growth in cities and their surrounding regions to improve the quality of life for all citizens. A CDS helps cities integrate a strategic development approach and a long-term perspective into their urban planning («About CDS», citiesalliance.org, 20I2).
\end{abstract}

La SDGS s'est tenue, pour sa première phase, qui sera particulièrement examinée ici, entre 2002 et 2006. L'objectif était d'élaborer une vision stratégique de Sfax et de sa région (gouvernorat de Sfax) à moyen terme (horizon 2016) et, dans une deuxième étape, à long terme (horizon 2030). Le processus d'élaboration a été organisé en quatre phases : diagnostic stratégique (une année); perspectives de développement et priorisation, deux tâches menées de front pendant plus d'une année (fin 2003 à 2005); élaboration de la vision stratégique (2006). Même si le territoire concerne les sept communes de l'agglomération, la stratégie a étendu son initiative sur l'ensemble du gouvernorat. L'élaboration de la SDGS à travers un processus relativement ouvert, critique et débouchant sur un diagnostic et des propositions partagés a été jugé comme une réussite par de nombreux participants et par les organisations internationales impliquées en tant que bailleurs de fonds. L'expérience sfaxienne a de ce fait été mise en avant par l'association Medcités et vantée comme un exemple de bonne pratique ${ }^{5}$.

\footnotetext{
${ }^{5}$ "Les éléments de la réussite de la SDGS I \& II sont :

- l'appropriation de la Stratégie par les élus des sept communes ;

- l'existence d'une certaine continuité au niveau des membres du comité de pilotage ;

- la prise en compte dans la stratégie des actions prévues par les documents d'urbanisme ;

- la capacité de lobbying des membres du comité de pilotage auprès des bailleurs de fonds internationaux » (Medcités, 2011 : 59).
} 
Tableau I

Bilan des actions projetées dans la SDGS I (élaboration : T. Megdiche d’après les documents SDGS I)

\begin{tabular}{|c|c|c|c|c|}
\hline $\begin{array}{l}\text { Domaine } \\
\text { d'action }\end{array}$ & Actions projetées & $\begin{array}{l}\text { Actions } \\
\text { entreprises et } \\
\text { achevées }\end{array}$ & $\begin{array}{l}\text { Actions } \\
\text { entreprises et } \\
\text { inachevées }\end{array}$ & $\begin{array}{l}\text { Actions } \\
\text { projetées et non } \\
\text { mises en œuvre }\end{array}$ \\
\hline \multirow{6}{*}{$\begin{array}{l}\text { Développement } \\
\text { économique }\end{array}$} & $\begin{array}{l}\text { Poursuite de la mise en place du } \\
\text { technopole Informatique -TIC }\end{array}$ & & $\mathrm{X}$ & \\
\hline & $\begin{array}{l}\text { Création d'un technopole } \\
\text { agroalimentaire }\end{array}$ & & & $\mathrm{X}$ \\
\hline & $\begin{array}{l}\text { Création d'un pôle de } \\
\text { biotechnologie }\end{array}$ & & & $\mathrm{X}$ \\
\hline & Création d'un pôle de santé & & & $\mathrm{X}$ \\
\hline & $\begin{array}{l}\text { Développement d'un pôle } \\
\text { touristique et de loisirs }\end{array}$ & & & $\mathrm{X}$ \\
\hline & $\begin{array}{l}\text { Création d'un Parc d'Activités } \\
\text { Économique à vocation } \\
\text { commerciale }\end{array}$ & & & $\mathrm{x}$ \\
\hline \multirow{6}{*}{$\begin{array}{l}\text { Renforcement } \\
\text { des } \\
\text { infrastructures } \\
\text { de base }\end{array}$} & $\begin{array}{l}\text { Renforcement des infrastructures } \\
\text { et services d'appui à l'économie }\end{array}$ & & & $\mathrm{X}$ \\
\hline & Mise à niveau du port & & & $X$ \\
\hline & $\begin{array}{l}\text { Extension et mise à niveau de } \\
\text { l'aéroport }\end{array}$ & $\mathrm{X}$ & & \\
\hline & $\begin{array}{l}\text { Création d'une plate-forme } \\
\text { logistique }\end{array}$ & & & $x$ \\
\hline & $\begin{array}{l}\text { Mise en place de grandes } \\
\text { infrastructures d'appui aux } \\
\text { activités socioculturelles }\end{array}$ & & & $\mathrm{X}$ \\
\hline & $\begin{array}{l}\text { Création d'un mode de transport } \\
\text { en site propre }\end{array}$ & & & $\mathrm{X}$ \\
\hline \multirow{2}{*}{$\begin{array}{l}\text { Dépollution et } \\
\text { amélioration du } \\
\text { cadre de vie }\end{array}$} & Dépollution du Grand Sfax & & $x$ & \\
\hline & $\begin{array}{l}\text { Préservation des ressources } \\
\text { naturelles }\end{array}$ & & & $\mathrm{X}$ \\
\hline \multirow{2}{*}{$\begin{array}{l}\text { Aménagement } \\
\text { urbain dans une } \\
\text { perspective } \\
\text { métropolitaine }\end{array}$} & $\begin{array}{l}\text { Promotion d'un modèle de } \\
\text { développement urbain littoral }\end{array}$ & & & $x$ \\
\hline & $\begin{array}{l}\text { Aménagement et valorisation du } \\
\text { littoral sud }\end{array}$ & & $\mathrm{X}$ & \\
\hline \multirow{2}{*}{$\begin{array}{l}\text { Institutionnalisat } \\
\text { ion du cadre } \\
\text { métropolitain }\end{array}$} & $\begin{array}{l}\text { Création d'un observatoire de } \\
\text { développement du Grand Sfax }\end{array}$ & & $\mathrm{X}$ & \\
\hline & $\begin{array}{l}\text { Création d'une structure } \\
\text { intercommunale }\end{array}$ & & & $\mathrm{x}$ \\
\hline \multicolumn{2}{|c|}{$\begin{array}{l}\text { Création d'un festival international de musique } \\
\text { méditerranéenne }\end{array}$} & & & $x$ \\
\hline
\end{tabular}


L'examen de ce processus inclura trois points: les origines du projet, qui mettent en évidence le rôle moteur des bailleurs de fonds par rapport aux autorités nationales et locales, qui a permis la constitution d'une arène de débat regroupant une diversité inédite de participants en Tunisie ; les modes de travail collectif, qui montrent un apprentissage de la collaboration et l'acquisition de nouveaux outils cognitifs ; l'argumentation mobilisée dans le projet, qui articule de vigoureuses revendications fondées sur une spécificité urbaine locale et une inscription dans la continuité des politiques étatiques.

\section{I Le rôle d'initiateur des bailleurs de fonds dans la mise en place d'un cercle participatif à l'échelle locale}

Le lancement de la SDGS est une initiative du programme de gestion urbaine (PGU), financé par le Centre des Nations Unies pour les établissements humains $(\mathrm{CNUEH})^{6}$, qui avait initié en 2000 à Tunis une initiative similaire, dont le bilan a été considéré comme mitigé. En effet, malgré les déclarations officielles, le processus participatif, élément central de la SDVT, avait été réalisé « en vase clos dans l'indifférence totale des autorités locales et du secteur privé » ainsi que de la société civile, et marqué par des « contraintes institutionnelles [...] et méthodologiques » (Medcités, 201I : 58). Pour les observateurs, il était resté « un exercice d'expert», selon les mots d'une consultante impliquée dans son organisation (Gafsi, 20ll). Les institutions tunisiennes de tutelle de ce projet, la Fédération nationale des villes tunisiennes (FNVT) et son partenaire l'Association tunisienne des urbanistes (ATU), étaient sous la pression du CNUEH et de quelques autres bailleurs ${ }^{7}$, qui n'ont accepté de financer le processus qu'à la condition d'une véritable participation. Un fonctionnaire du ministère de Développement, originaire de Sfax, parvint à convaincre le maire de la ville, un haut cadre de l'administration, de tenter l'expérience. Une première originalité consiste dans l'agrégation au processus des six maires des petites communes périphériques de l'agglomération, un exemple extrêmement rare de coopération intercommunale obtenue suite à l'insistance du bailleur de fonds.

\footnotetext{
${ }^{6}$ Le CNUEH est connu en anglais comme UN-Habitat.

${ }^{7}$ Ces bailleurs étaient : Medcités, la Banque mondiale, le CNUEH par l'intermédiaire du Programme de gestion urbaine, l'AFD, le Fonds Mondial de l'Environnement, le Députaccio de Barcelone, la GTZ (agence de coopération et de développement allemande) et Cities Alliance, pour un financement total, pour la phase I, de 900.000 dinars.
}

L'équipe du projet était formée par les sept maires du Grand Sfax, la FNVT, l'ATU, les chefs d'équipes et les consultants. Sur un plan méthodologique, six commissions ont été créées: développement urbain et gestion municipale, transport et circulation, environnement, jeunesse, développement économique et social, culture et communications et relations avec les médias. Les six commissions, présidées par un représentant des communes, étaient dirigées par des experts, et la majorité étaient formées d'universitaires de Sfax sans engagement politique connu. Chaque commission organisait ses propres réunions et invitait sans restriction tous les acteurs du domaine étudié. Des réunions générales regroupant les différentes commissions ont été organisées. Deux membres du conseil municipal de Sfax, universitaires, étaient particulièrement chargés d'organiser le processus et ils ont convaincu d'autres universitaires d'y participer. De même, plusieurs ONG ont rejoint les réunions. Si certaines étaient clairement affiliées au régime (l'Union des femmes tunisiennes par exemple), d'autres, animées par des universitaires et des professions libérales comme l'APNES (Association de protection de la nature et de l'environnement de Sfax) et l'Association des amis des oiseaux, étaient indépendantes.

\subsection{Le travail collectif : un apprentissage de la collaboration et l'acquisition de nouveaux outils cognitifs}

L'élaboration de la SDGS a reposé sur un processus évolutif où l'apprentissage du travail collectif s'est substitué progressivement à la méfiance initiale. Dans ce cadre, les coopérations se sont nouées à la fois entre les acteurs locaux et les experts internationaux, mais aussi entre les représentants de l'administration locale et centrale liés au parti au pouvoir et les participants issus de la société civile (associations, universitaires en particulier). Enfin, la participation a été voulue assez large en direction du public.

Dans le processus, les représentants des bailleurs de fonds ont pu jouer un rôle de catalyseur grâce aux outils intellectuels qu'ils ont apporté et mis à la disposition des participants. Ainsi, les experts de Medcités ont aidé les participants par la méthodologie de mise en place des fiches projets pour les rendre bancables. Les subventions ont concerné le financement des études. Ainsi, l'étude de faisabilité du tramway a été initiée avec l'appui de la Banque Européenne d'Investissement. De même, un Agenda 2I pour la médina de Sfax a été financé grâce à une 
Tableau 2

Études réalisées dans le cadre de la SDGS, phase II (2008-20 I0)'

\begin{tabular}{|l|l|}
\hline Étude & Financement \\
\hline 1- Zones urbaines populaires & $\begin{array}{l}\text { Banque Mondiale } \\
60000 \text { dinars }\end{array}$ \\
\hline $\begin{array}{l}\text { 2- Observatoire du } \\
\text { Développement Durable du } \\
\text { Grand Sfax }\end{array}$ & $\begin{array}{l}\text {-Deputacio de Barcelone } \\
\text {-Med Cities }\end{array}$ \\
\hline $\begin{array}{l}\text { 3- Promotion de l'emploi } \\
\text { 4- Stratégie de } \\
\text { Développement économique } \\
\text { local }\end{array}$ & $\begin{array}{l}\text { Banque Mondiale } \\
65000 \text { dinars } \\
65000 \text { dinars }\end{array}$ \\
\hline \begin{tabular}{l} 
5- Transport \\
\hline
\end{tabular} & $\begin{array}{l}\text { Ministère du Transport } \\
50000 \text { dinars }\end{array}$ \\
\hline
\end{tabular}

demande effectuée dans ce cadre. Dans le prolongement de cette mobilisation, durant la phase II du processus entre 2008 et 2010 , cinq études ont été réalisées grâce au soutien renouvelé de certains bailleurs de fonds. Elles ont concerné le développement économique local dans son ensemble, la création d'emplois et la résorption du chômage, notamment des jeunes, des femmes et des non diplômés, l'intégration urbaine des zones populaires les plus défavorisées afin de renforcer la cohésion sociale au niveau de ces zones et celui de l'agglomération, l'amélioration de la mobilité et des conditions du transport en commun et la création d'un Observatoire du Développement du grand Sfax. Certains projets ont résulté de l'initiative propre de l'équipe d'experts locaux de la SDGS. Ce fut le cas du projet d'observatoire urbain, proposé par les universitaires locaux. D'autres idées ont été soufflées ultérieurement par les bailleurs de fond internationaux, comme la nécessité d'une étude au sujet des quartiers populaires. Le tableau 2 ci-dessous rend compte des projets financés au titre de la SDGS II, sachant que d'autres projets identifiés dans la première phase ont été inclus dans une «banque de projets », permettant aux bailleurs de fonds de les sélectionner et d'y apporter des financements en fonction de leurs propres priorités, recoupant alors celles de la SDGS ${ }^{8}$.

L'interaction entre acteurs issus de l'administration et ceux de la société civile s'est traduite par le partage progressif d'un langage commun. Même si l'acteur public était toujours présent dans les réunions des commissions et des séances plénières, les experts et les universitaires, ainsi que les ONG à l'image de l'association de protection de la nature et de l'environnement, se sont sentis libres d'exprimer leurs opinions et critiques sur les choix de l'acteur public. Ainsi, ils sont devenus progressivement plus constructifs, voyant que leurs critiques étaient entendues. De même, lors des premières rencontres, les représentants régionaux de l'administration et les directeurs des services publics étaient embarrassés et ne savaient quel type d'attitude ils devaient adopter. Ils venaient le plus souvent avec les directives de leur administration centrale, limitaient les sujets de leur intervention et avaient peur de

\footnotetext{
${ }^{8}$ Notamment l'aménagement durable du littoral, intégré dans le programme de gestion intégrée, voir infra.
} 
s'exprimer librement. Certains représentants du parti RCD s'invitaient parfois en leur qualité pour un rappel à l'ordre de leurs troupes et pour faire passer un discours politique. Un architecte de la commune de Sfax fut ainsi malmené lors d'une réunion par un délégué et un élu RCD, parce qu'il avait osé parler de manque d'infrastructures, etc. Pourtant, il est arrivé à plusieurs reprises que le maire, censé être du régime car il représentait le RCD au pouvoir, adopte les points de vue des experts indépendants. Mais un dialogue se noua progressivement autour du thème de la marginalisation de Sfax (voir ci-dessous), autour duquel une large partie des participants, y compris ceux affiliés au pouvoir, se sont retrouvés. De même, les propositions élaborées dans la SDGS n'ont pas heurté les décideurs dans la mesure où ils ont gardé les choix préconisés par le Schéma directeur d'aménagement du Grand Sfax (SDAGS) de 1998.

La participation du grand public fut explicitement recherchée grâce à une stratégie affirmée de communication. Le lancement de la SDGS a été accompagné par l'édition de 20000 dépliants destinés aux acteurs économiques et culturels de la ville et aux citoyens et d'un film DVD en trois langues. La stratégie de communication a aussi inclus une identité visuelle largement déclinée sur divers supports, permettant plus de lisibilité et de sensibilisation médiatique à la stratégie (photo 2). De 2002 à 2005, la stratégie a réuni plus de 5000 participants, organisé 7I réunions des groupes de travail, 12 réunions de chaque comité du projet, etc. Des réunions grand public ont été aussi organisées dans les communes périphériques, autour de thèmes comme la pollution, la jeunesse, la réhabilitation des quartiers populaires, etc.

Ces réunions procédaient principalement d'une logique descendante d'information et de légitimation de la stratégie, mais elles donnèrent également lieu à une validation des thèmes proposés par les experts indépendants, tels que le problème de la pollution ou celui des espaces verts. Ainsi, ces réunions ont contribué à l'établissement du consensus. En revanche, elles n'ont pas réellement fait émerger de nouveaux enjeux ou propositions.

\subsection{Registre argumentaire des participants locaux : une réinscription dans l'histoire locale du développement et de l'aménagement}

Le diagnostic élaboré par la SDGS s'est largement structuré autour de l'idée d'une ville au potentiel de développement important, notamment en lien avec son milieu entrepreneurial très dynamique (Denieuil, 2000). Mais le constat était surtout celui d'une perte de souffle économique de la ville, ressentie depuis la fin des années 1970, et du désengagement précoce de l'État (la ville et sa région n'ont reçu que $4 \%$ des

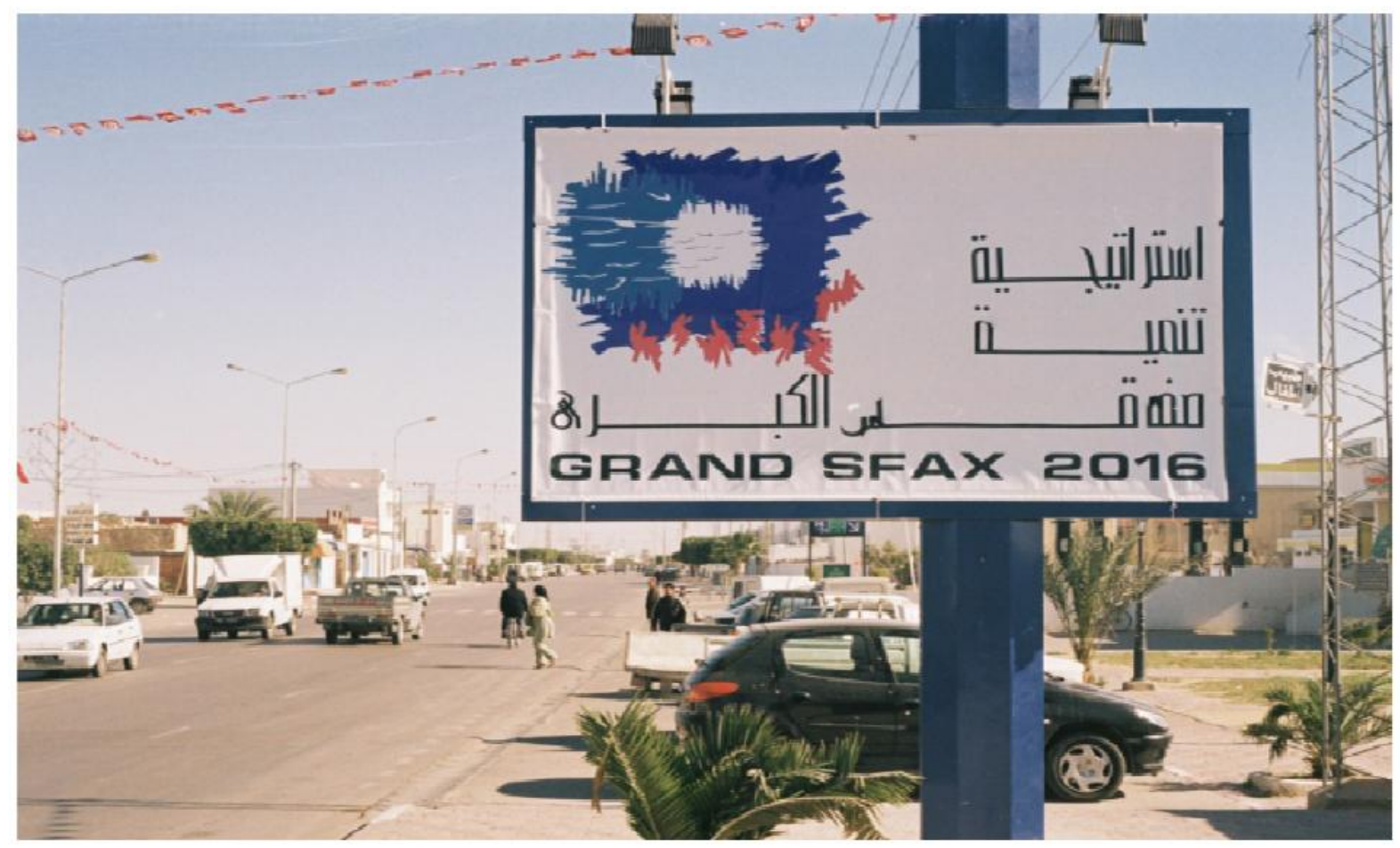

Fig. 2 - logo de la Stratégie de développement du Grand Sfax 
investissements publics entre 1962 et 1969 , pour 10\% de la population du pays). Derrière ces faits, les participants à la SDGS relèvent l'existence d'un discours anti-Sfax (parfois décrite comme un village, une société fermée, etc.) de la part de l'acteur public et l'interprètent comme une forme de marginalisation de la ville et de sa région à la source d'une véritable frustration sfaxienne. Ce sentiment n'était pas uniquement celui des critiques du régime : il était partagé par des gens proches du régime et par des élus lorsqu'ils parlaient dans des cercles restreints. Certains hommes d'affaire (industriels), de même que l'ancien maire de la ville, aujourd'hui président de l'APNES, médecin promu ministre de la Jeunesse, ont toujours émis des critiques virulentes contre le régime, mais aussi contre les élus et les acteurs publics sfaxiens qui ne défendaient pas leur ville.

Les premières réunions de la SDGS se sont transformées en plaidoyer pour la ville : ville polluée, sous-équipée, avec une absence problématique d'espaces de loisir et de détente, lieu de concentration des flux d'exode de l'intérieur, que l'on accuse d'être « injustement » renvoyés du Sahel touristique voisin vers Sfax, etc. Le départ des capitaux, des hommes de culture et du savoir vers Tunis ou vers l'étranger est également regretté et motive la formulation du schéma de développement. Les solutions mises en avant s'inscrivent dans la droite ligne de ce consensus local, autour de la reconquête du littoral et de la réaffirmation d'une identité de ville méditerranéenne. Trois grandes priorités ont été dégagées (Charfi, 2005) :

- le développement économique et urbain par la mise en place des grands projets structurants (pôle technologique, plateforme stratégique de transport avec la dynamisation du port, de l'aéroport, de l'autoroute, développement des industries et des activités innovantes, recentrage de l'urbanisation). En appui à ces projets, la SDGS prévoit la mise en place d'un observatoire urbain ;

- la réconciliation de Sfax avec son littoral a figuré parmi les grands projets que la stratégie a développé ; il s'agit du projet Taparura de réhabilitation du littoral nord, de la réhabilitation du littoral sud après la fermeture annoncée de l'usine des phosphates SIAPE et du transfert des sources de pollution (décharge publique, dépôt de margines, savonneries, etc.) ;

- la dimension sociale a été aussi développée. Les grandes orientations sont la lutte contre l'exclusion socio-spatiale et la marginalisation à travers la réhabilitation des quartiers populaires, le développement des équipements et infrastructures, notamment de transport avec un projet de métro léger, le développement de l'arrière-pays, la promotion du tourisme durable, etc.

- Les projets figurant dans la stratégie sont pour une large part des projets déjà existants mais non réalisés, figurant notamment dans le SDAGS de 1998, mais aussi parfois dans des programmes antérieurs. En validant la stratégie, le pouvoir ne s'est donc pas déjugé. Celle-ci a plutôt confirmé la justesse de ses vues. La section suivante a pour objet d'analyser, dans ce cadre marqué par la continuité et par des rapports de force inchangés, la manière dont les acteurs locaux ont néanmoins pu pousser des projets innovants.

\subsection{La reconquête de la façade littorale de Sfax : entre continuités et innovations des logiques d'élaboration des projets de développement durable}

La façade littorale sfaxienne a été vouée, dès la fin des années 1940, à des industries et équipements polluants, dont les résidus occupent de vastes étendues aux portes de la ville: dépôts de phosphogypse, décharge municipale, dépôt de margine (résidus de l'extraction d'huile d'olive). Les remblais destinés à l'extension du port et les infrastructures de transport, notamment le chemin de fer, contribuent à couper la ville de la mer, interdisant les pratiques de loisir et de détente (interdiction de la baignade à partir de 1978).

Dans ce contexte, deux actions préconisées par la SDGS peuvent servir d'exemples pour analyser dans quelle mesure les logiques d'élaboration et le contenu des projets placés sous la qualification du développement durable sont transformés. Le premier exemple, dénommé Taparura, consiste en un ambitieux projet de réhabilitation du littoral nord de la ville, très dégradé (Megdiche, 20I2). La poursuite de ce projet, cohérent avec la stratégie, reste cependant complètement du ressort de l'État et échappe aux acteurs locaux. En contrepartie, il est toutefois nécessaire de remarquer que la dynamique participation-revendication-négociation engagée a également abouti, dans un deuxième temps, à des projets de transformation plus importants, plus novateurs par rapport au répertoire des actions déjà engagées, et qui illustrent la capacité du processus enclenché à faire évoluer le contenu de l'action publique. C'est ce que montre le projet de réhabilitation du littoral sud (Megdiche, 2010). 


\subsection{Taparura : la permanence d'un développement imaginé et contrôlé par l'État}

La grande opération de réhabilitation du littoral nord de Sfax n'a guère été débattue dans le cadre de la SDGS, probablement en raison de son caractère de « projet présidentiel ». Cette opération, dont la zone d'intervention s'étend sur 420 hectares gagnés sur la mer, doit permettre d'assainir six kilomètres de plages, de créer des zones touristiques, résidentielles, d'équipements publics et des espaces verts. L'idée du projet remonte au début des années 1980. Au moment où Tunis décidait d'assainir sa lagune et de transformer ses berges en pôle urbain moderne, Sfax ambitionna aussi de réaliser son « projet du siècle » en dépolluant le littoral nord et en gagnant des terrains sur la mer. En 1985, à la suite de la mobilisation de certains acteurs de la ville, l'État crée une Société d'études et d'aménagement des côtes nord de Sfax. La fermeture, en 1992, de l'usine de traitement des phosphates NPK, responsable de la pollution du littoral nord, a ouvert la voie à sa réhabilitation et à l'avancement des études pour la réalisation du projet. Mais il a fallu plus de vingt ans pour que les travaux de dépollution et de remblai commencent, grâce à la signature, en juillet 2004, d'une convention conclue entre le gouvernement tunisien et la Banque Européenne d'Investissement, relative au prêt de 34 millions d'euros accordé au gouvernement tunisien pour la contribution au financement du projet « assainissement du site Taparura »".

Au début des années 2000, lors de l'élaboration de la SDGS, le projet n'avait pas encore décollé. Il était clair, dès le début, que cette opération nécessiterait beaucoup d'études et beaucoup de moyens pour être réalisée. Mais l'avancement du projet a été particulièrement lent, surtout aux yeux des habitants et des acteurs locaux. La première décennie a été consacrée à la réalisation de nombreuses études pour s'assurer de la faisabilité technique du projet et de sa rentabilité économique, ainsi que de différentes études d'impact sur l'environnement, de schémas d'urbanisme et de financement. En outre, il a été décidé en 1997 de réaliser le projet par étapes, en commençant par la composante dépollution, qui concerne une zone d'intervention limitée à 260 hectares.

En fait, les grandes décisions concernant ce « projet présidentiel » étaient prises en conseil des ministres, présidé par le président de la République : fermeture de l'usine polluante NPK, réalisation du projet en deux étapes, délimitation de la zone

\footnotetext{
${ }^{9}$ Sur le processus d'aménagement de ce projet, voir Megdiche 2012.
}

d'intervention, etc. Dans le contexte tunisien, l'exemple de Taparura est assez représentatif de la gouvernance des grands projets mis en œuvre au cours des deux dernières décennies. En 2005, l'État a créé une «commission nationale des grands projets » pour la supervision et le suivi; elle est rattachée directement à la présidence de la République ${ }^{10}$. La réalisation de ces projets a été engagée par des conventions entre le gouvernement et les investisseurs et avalisée par le vote du parlement. Ces projets étaient annoncés et conclus dans des conditions peu transparentes. La gouvernance du projet Taparura est donc marquée par la centralisation du pouvoir de décision et ne diffère pas de ce que d'autres études ont signalé au sujet de la gestion de la ville de Tunis, marquée par «la réduction de l'autonomie et de l'autorité des ministères, en lien avec la tendance à la présidentialisation de l'aménagement» (Barthel, 2006 : 56).

Le contrôle de l'État central sur ce projet est total. La Société d'Études et d'Aménagement des Côtes Nord de la ville de Sfax, ou SEACNVS, en charge du projet, est une société anonyme à participation publique et sous tutelle du ministère de l'Équipement, de l'Habitat et de l'Aménagement du Territoire. Ses prérogatives sont limitées: elle est chargée uniquement des études et de la réalisation des travaux du projet. L'État contrôle aussi le capital de la société puisque les principaux actionnaires sont des organismes publics ou parapublics, des collectivités publiques et des banques publiques et semi privées. C'est également l'État qui a contracté des prêts auprès des investisseurs étrangers, le financement du projet étant en grande partie assuré par des organismes étrangers. Sur le plan foncier, c'est aussi l'État qui est propriétaire des terrains gagnés sur la mer. Après l'achèvement des travaux de dépollution et de remblai, la zone concernée a été déclassée du domaine public maritime par un décret, pour être incorporée au domaine privé de l'État.

Vanté au nom de l'écologie et du développement local, le projet Taparura reste à l'heure actuelle en attente d'un véritable programme d'urbanisme. On peut constater que les acteurs locaux dans l'administration, les collectivités locales et la société civile sont marginalisés dans le processus décisionnel. La participation locale se réduit à l'implication des experts et chercheurs universitaires, notamment ceux de l'École Nationale des Ingénieurs de Sfax, qui ont souvent été associés à l'élaboration des études techniques.

\footnotetext{
${ }^{10}$ Cette commission a été dissoute après le 14 janvier $20 \mathrm{II}$
} 


\subsection{Le littoral Sud et la relocalisation des nuisances : des projets de développement durable résultant de la dynamique participative locale}

Le littoral sud de Sfax présente de multiples sources de nuisances et renferme de nombreux sites pollués comme le dépôt de phosphogypse, les bassins de stockage des margines, la décharge municipale non contrôlée et la station d'épuration. C'est pour cette raison que la Stratégie de Développement du Grand Sfax a retenu dans le cadre de l'axe stratégique " Dépollution et amélioration du cadre de vie » un projet structurant sur « la dépollution et réhabilitation des côtes Sud de Sfax ». Ce projet implique des actions correctrices par un programme urbanistique spécifique, préconisant notamment la valorisation de la zone humide de Thyna et de son site archéologique. Sous l'appellation de schéma de « gestion intégrée de la zone côtière sud de Sfax »(GIZCS), réalisé entre 2006 et 2008, les objectifs de ce projet consistent en la préservation du littoral Sud du Grand Sfax des méfaits des pollutions diverses, l'amélioration de la qualité de vie et des conditions sanitaires de la population et la réalisation d'une complémentarité fonctionnelle et paysagère des ailes Nord et Sud de la façade maritime du Grand Sfax, la réussite du projet Taparura au Nord restant tributaire du devenir de la côte Sud.

Le lancement de l'étude a bénéficié des programmes promouvant le développement durable dans lesquels l'État tunisien s'est engagé depuis les années 1990, notamment le Short and Middle Action Programme -Tunisie (SMAP-III) de la Commission Européenne. Ce programme d'actions prioritaires à court et moyen terme pour l'environnement découle de la Déclaration de Barcelone de 1995 et de la Conférence Euro-Méditerranéenne d'Helsinki de 1997. Les trois générations de ce programme (SMAP I (1999), SMAP II (200I) et SMAP III (2005)'I) visent à soutenir le développement durable et particulièrement les activités environnementales dans la région côtière de la Méditerranée. Plusieurs domaines prioritaires d'intervention y sont prévus, dont la Gestion Intégrée des Zones Côtières (GIZC). Le littoral sud de Sfax a été choisi avec la région rurale de Kroumirie et Mogods dans le Nord de la Tunisie, pour l'élaboration de stratégies de gestion intégrée des zones côtières

\footnotetext{
II Voir

http://www.medcities.org/docs/Smap_english_maxibrochure.pdf, consulté le 17 septembre 2012 .
}

$\left(G I Z C^{12}\right)$ afin de servir d'exemples pour le développement d'un plan national de gestion intégrée du littoral.

La décision d’intégrer Sfax à ce programme a été prise dans le sillage de la SDGS. Elle a émergé à partir d'une initiative locale, notamment des associations de défenses de l'environnement, mise en avant dans le programme d'action avec l'appui des organismes internationaux, dont l'Union européenne. Ce projet constitue en réalité une réappropriation d'orientations visant à la reconquête des zones polluées existant dans le SDAGS de 1998, mais qui étaient restées lettre morte. L'originalité de cette action réside donc moins dans son contenu que dans sa reprise et son partage par les divers intervenants.

L'étude a été réalisée dans le cadre d'un partenariat entre les sept communes du Grand Sfax, l'Université de Sfax, la Société d'Études et d'Aménagement des Côtes Nord de la Ville de Sfax (projet Taparura), l'Agence de Protection et d'Aménagement du Littoral, avec l'appui de l'Union Européenne, Med Cités, le Fonds Mondial pour la Nature (WWF) et le Programme des Nations Unies pour le Développement (PNUD) en Tunisie. À l'instar de la SDGS, la conduite des diverses étapes du projet a associé les principaux acteurs de la zone, et plus particulièrement les administrations, les représentants de la Société Industrielle d'Acide Phosphorique et d'Engrais (SIAPE), responsables du dépôt de phosphogypses, et ceux de la Compagnie Tunisienne des Salines de Tunisie (COTUSAL). Les travaux commencés en janvier 2006 et finalisés en mai 2008 ont suivi une approche similaire à celle de la SDGS. Ils ont permis de faire un diagnostic du littoral et d'identifier des projets visant la dépollution et la valorisation de cette zone, notamment par la réalisation d'un plan d'actions prioritaires comprenant la fermeture de l'usine de la SIAPE, la réhabilitation du dépôt de phosphogypse, l'intégration urbaine des salines et la promotion du « tourisme durable » et de la « pêche durable ».

Cet exemple montre que le développement durable et ses dispositifs partenariaux (international/national/local) permettent de remobiliser des idées viables existantes, mais en les réhiérarchisant (en en faisant des priorités) grâce à l'engagement d'une dynamique qui permet l'obtention d'un accord.

\footnotetext{
${ }^{12}$ Voir le document présentant la Stratégie européenne de gestion intégrée des zones côtières :

http://ec.europa.eu/environment/iczm/home.htm, consulté le 17 septembre 2012.
} 
Mais cette approche a un caractère élitiste et techniciste. Elle s'est limitée à impliquer les universitaires, les experts, les représentants des administrations et des associations (principalement l'APNES). En outre, ce processus ne s'appuie pas sur un dispositif de gouvernance urbaine solide. Les collectivités locales n'ont aucun pouvoir de décision et ne disposent pas de ressources financières pour réaliser les projets. De manière générale, le pouvoir de décision échappe totalement aux acteurs locaux.

En outre, l'adoption de ces propositions par le président Ben Ali en septembre 2008, dans un contexte qui était celui de la campagne menée en faveur de sa réélection, conduit à considérer cette décision comme un gage donné aux associations mobilisées sur ces questions, dans une quête de légitimité et de reconnaissance du «modernisme » présidentiel.

La SDGS et ses suites peuvent se lire comme un processus d'élaboration de projets de développement alternatif aux modes habituels en usage en Tunisie, marqués par le centralisme et par la logique autoritaire et clientéliste dans la prise de décision. Sur ce terrain, on constate l'émergence d'une logique d'action se revendiquant du développement durable, fortement soucieuse de résoudre les graves problèmes environnementaux de la ville de Sfax, d'identifier de nouvelles logiques de développement économique, et appuyée sur des forces locales extérieures à l'administration et au parti, notamment celles issues de la société civile. Cette émergence est rendue possible par la conjonction de trois éléments : un régime qui, tout en gardant le contrôle des leviers de pouvoir, cherche à utiliser la thématique du développement durable pour sa légitimation; des bailleurs de fonds internationaux qui posent comme condition à leurs apports financiers et à leur appui institutionnel et technique, l'adoption de nouvelles logiques d'action; et la mobilisation d'une élite locale diversifiée dans ses allégeances politiques et parfois éloignée du régime, mais fortement désireuse de s'impliquer dans une autre logique de développement dans son contenu et dans ses modes d'élaboration. II est clair que, même s'il apporte de la fraîcheur dans l'aménagement urbain tunisien et suscite à ce titre des appréciations favorables des techniciens locaux (Gafsi, 20II) et des bailleurs de fonds (Medcités, 20lI), ce processus ne transforme pas fondamentalement les rapports de force et les logiques de décision.

\section{LE DÉVELOPPEMENT URBAIN DURABLE DANS LE CONTEXTE RÉVOLUTIONNAIRE TUNISIEN : LA CONTROVERSE AUTOUR DE LA DÉLOCALISATION DE LA SIAPE}

L'objectif de cette dernière section est de revenir sur la dynamique engagée par le SDGS à la lumière des changements politiques résultant de la révolution tunisienne de 20II, afin de s'interroger sur les actions entreprises au nom du développement urbain durable dans un contexte où, d'un coup, les arènes de discussion sur les politiques urbaines sont très largement ouvertes à de nouvelles voix et à de nouveaux acteurs. La manière même de formuler les enjeux transforme les objectifs considérés comme souhaitables. Le consensus précédemment obtenu est ainsi contesté et la légitimité même de la notion de développement durable est mise en cause par une partie du public. Ce point est analysé à travers la controverse qui entoure le projet de délocalisation de la SIAPE, l'usine polluante emblématique de la dégradation de l'environnement sfaxien par ses fumées et ses dépôts de phosphogypse.

Sous la pression des mobilisations populaires qui ont conduit au renversement du régime de Ben Ali, les gouvernements qui se succèdent depuis le 14 janvier $201 \mathrm{l}$ ont mis en avant deux priorités dans l'action publique : le développement des régions intérieures, dont la négligence par le précédent régime est vue comme une cause essentielle de la révolution, et la création et la défense de l'emploi, alors que le chômage, déjà élevé, souffre des incertitudes économiques de la transition. C'est ainsi que le gouvernorat de transition a décidé l'affectation de $80 \%$ des fonds prévus par la loi des finances $201 \mathrm{I}$ aux régions intérieures et de $20 \%$ seulement de ces fonds aux régions littorales. La même démarche a été adoptée par l'actuel gouvernement: dans la loi de finances complémentaire de $2012,70 \%$ des ressources sont orientées vers le développement régional, principalement les gouvernorats de l'intérieur (Bennasr, 2012). Par ailleurs, les entreprises et administrations publiques recrutent massivement, régularisant des emplois auparavant précaires et embauchant de nouveaux salariés, notamment issus de la jeunesse. De nombreux mouvements sociaux des habitants des régions défavorisées ou des secteurs urbains pauvres occupent l'espace public et exercent des pressions sur les entreprises, privées et plus encore publiques, pour défendre les emplois ou 
obtenir des embauches. Les syndicalistes de I'UGTT, qui soutiennent largement ces actions et s'y impliquent parfois, apparaissent comme une puissante force politique, auréolée d'actions de résistance au régime dans le bassin de Gafsa dès 2008 (Chouikha et Geisser, 2010) ${ }^{13}$.

Dans ce contexte, la question du développement durable est reléguée à l'arrière-plan des préoccupations politiques, en particulier dans son volet environnemental. Pour certains observateurs, un inventaire critique de la notion même de développement durable est à effectuer (par ex. Gafsi, 20II). En effet, il est noté que cette thématique a constitué un outil de légitimation du régime, au plan interne comme au plan international. Parfois, les politiques ont masqué des malversations ou des réalités moins avouables (occupation illégale de terrains protégés, etc. $)^{14}$.

La controverse autour du transfert prévu de la SIAPE vers la ville de La Skhrira, à une soixantaine de $\mathrm{km}$ au sud de Sfax, illustre ce changement d'état d'esprit. Prise en 2008 par Ben Ali, la décision de délocalisation aurait dû être mise en œuvre en $201 \mathrm{l}$. Elle fait aujourd'hui l'objet d'une polémique qui prend parfois un tour violent. En effet, des représentants du syndicat des employés de l'entreprise contestent ce projet avec virulence, au nom des droits des travailleurs pour qui le déplacement de l'usine rendrait les conditions de travail très pénibles. Leur argumentaire voit dans ce projet une action opportuniste de Ben Ali pour faire valoir sa légitimité et son modernisme à la veille de sa réélection, et assimile même les associations environnementales qui ont œuvré à la prise de cette décision à des complices du régime, tout en relativisant l'impact des pollutions atmosphériques par rapport à d'autres émissions liées au transport par exemple ${ }^{15}$.

Pour leur part, les tenants de la délocalisation ont lancé une grande campagne en direction du public, en mobilisant notamment les habitants des secteurs les plus exposés à la pollution. Le débat déborde largement au-delà des milieux précédemment impliqués et occupe l'espace public via les réseaux

\footnotetext{
${ }^{13}$ Même si la direction du syndicat est restée fidèle au régime, les sections de base se sont souvent montrées indociles. À Gafsa, le syndicat a joué un rôle majeur dans les troubles qui ont éclaté en 2008 et qui sont considérés comme un élément annonciateur de la révolution de décembre 2010-janvier 201 I.

${ }^{14}$ Point analysé plus en détail dans Verdeil (2011).

${ }^{15}$ Voir par exemple la discussion sur

http://www.sfaxonline.com/sfax-developpement/sfax-

environnement//812-------qq-, consulté le 17 septembre 2012.
}

sociaux ${ }^{16}$ et lors de conférences et manifestations organisées en ville. Lors de l'une d'elles, en mai 2012, le président provisoire Moncef Marzouki a d'ailleurs apporté son soutien au projet (bien qu'il ne dispose pas de pouvoir décisionnel à ce sujet).

La controverse illustre le poids du contexte politique au sens large sur la structuration des débats autour du développement urbain durable et la centralité qu'y tiennent les visions et les projets élaborés en son nom. Alors que les modalités d'élaboration de ces projets apparaissaient relativement innovantes, le nouveau contexte les discrédite ou du moins les relègue à l'arrière-plan en mettant au centre la question sociale (Hibou 20l I).

\section{CONCLUSION}

Dans cet article, nous nous sommes attachés à comprendre la construction d'une politique de développement urbain durable à Sfax en Tunisie, dans un contexte politique marqué par l'autoritarisme et par la centralisation. La SDGS et ses suites constituent un exemple de politiques considérées comme un succès par les bailleurs de fonds ainsi que par de nombreux professionnels locaux, qui y voient une manière originale de sortir de modèles imposés et d'approches descendantes et de prendre véritablement en compte les enjeux du territoire local, en particulier du point de vue de l'environnement, et en faisant émerger une vision partagée des acteurs du développement local.

Notre analyse a montré l'existence d'un processus localisé de revendication, d'apprentissage et de coproduction d'une vision locale de développement urbain durable, impliquant effectivement une pluralité d'acteurs locaux, y compris distants du régime. Ce processus a pu se déployer grâce à l'appui intellectuel et financier d'organisations internationales ellesmêmes fortement impliquées dans ce processus. Les instances nationales ont accompagné le processus, sans le bloquer, car d'une part, il ne remettait pas en cause les ni intérêts directs du régime ni sa légitimité. Au contraire même, l'une des caractéristiques de ce processus a été la remobilisation d'idées existantes mais délaissées. Un élément extrêmement déterminant a été le partage d'une perception de la ville de Sfax

\footnotetext{
${ }^{16}$ Voir notamment la campagne sur Facebook : Tous unis pour fermer la SIAPE et lever l'embargo sur Sfax,

http://www.facebook.com/event.php?eid=|7739027898 I873, (consulté le 6 juin 20I2)
} 
comme négligée et brimée, un sentiment dans lequel la dégradation environnementale a joué un rôle très important.

Toutefois, le fait de souligner la force de cette mobilisation locale dans l'élaboration de cette vision et dans la détermination d'un agenda d'actions partiellement mis en œuvre ne doit pas empêcher de voir les limites de ce processus, lié à la dépendance des acteurs locaux aux décisions réglementaires et financières des acteurs nationaux et internationaux. $\mathrm{Ce}$ fait est particulièrement souligné par le bouleversement du jeu politique avec la révolution tunisienne. Le développement urbain durable apparaît alors comme un registre d'action de l'ancien régime, et les acteurs locaux indépendants qui s'étaient efforcés de faire avancer certains dossiers se retrouvent en porte-à-faux avec la redéfinition des priorités vers les enjeux sociaux et d'équité territoriale, mis en avant par d'autres acteurs politiques. Ce fait rappelle que le développement urbain durable, même s'il peut faire l'objet d'une mobilisation locale, est fortement dépendant de ses modalités d'institutionnalisation initiale en contexte centralisé et autoritaire. Les sociétés arabes en bouleversement vont devoir se réapproprier les thèmes du développement durable, ce qui ne pourra se faire qu'en reconstruisant des visions partagées avec les segments les plus marginalisés de leurs populations.

\section{BIBLIOGRAPHIE}

BARTHEL, P.-A. (2006). Tunis en projet(s) : la fabrique d'une métropole au bord de l'eau, Rennes, Presses universitaires de Rennes, 206 p.

BARTHEL, P.-A. (2008a). "Faire la ville à Tunis sans les citadins? L'épreuve de la participation entre restrictions et irruptions dans le projet urbain $॥$. In Legros, O. (éd.), Participations citadines et action publique. Dakar, Rabat, Cotonou, Tunis, Jérusalem, Sanaa, Paris, ADELS, p. 15-48.

BARTHEL, P.-A. (2008b). " Faire du « grand projet » au Maghreb. L'exemple des fronts d'eau (Casablanca et Tunis) », Géocarrefour, vol. 83, no I, p. 25-34.

BARTHEL P.-A., V. CLERC et P. PHILIFERT (2013) « La « ville durable " précipitée dans le monde arabe : conservation d'un ordre ou conduite de changement de l'action urbaine ? ", Environnement urbain [En ligne], vol. 7, URL : http://www.vrm.ca/cyber-revues.asp.
BENNASR A. (20/2). «Le schéma d'aménagement du territoire national tunisien ou comment concilier compétitivité, efficacité et durabilité». In MESINI, B. (éd.), Aménagement durable des territoires méditerranéens, Marseille, Publications de l'Université de Provence, p. 49-58.

BERRY-CHIKHAOUI, I. et S. HAOUES-JOUVE (20II). «La fabrique du développement urbain durable à Marrakech: effets de contextes, interprétations et construction de l'Agenda 21 local». In BARTHEL, P.-A. et L. Zaki (éd.), Expérimenter la "ville durable » au sud de la Méditerranée. Chercheurs et professionnels en dialogues, La Tour-d'Aigues, Editions de l'Aube, p. 29I319.

CHABBI, M. (20I2). L'urbain en Tunisie. Processus et projets, Tunis, Nirvana, $220 \mathrm{p}$.

CHARFI, F. (2005). Stratégie de développement du Grand Sfax, Phase IV. Rapport de synthèse, non publié, Sfax, 109 p.

CHOUIKHA L. et V. GEISSER (2010). « Retour sur la révolte du bassin minier. Les cinq leçons politiques d'un conflit social inédit ", L'Année du Maghreb, no 6, p. $4 \mid 5-426$

CITIES ALLIANCE (20I2). "About CDS », citiesalliance.org [En ligne], URL: http://www.citiesalliance.org/aboutcds. [consulté le 16 mai 20I2]

DENIEUIL, P.-N. (2000). Les entrepreneurs du développement: L'ethno-industrialisation en Tunisie: la dynamique de Sfax, Paris, L'Harmattan, 208 p.

GAFSI, H. (2010). Histoire de l'évolution du domaine "Environnement en Tunisie » (Problèmes, acquis, défis) (Rapport final non publié), Tunis, GTZ, 34 p.

GAFSI, H. (20II). « Villes durables, un concept à revisiter dans le contexte de la révolution tunisienne? ", Présentation au séminaire Les premières expérimentations de la ville durable au Sud de la Méditerranée, Tunis-Sidi Bou Saïd, IRMC-CEDEJ, 19 avril 20I I.

HIBOU, B. (20II). « Le moment révolutionnaire tunisien en question : vers l'oubli du mouvement social? », Dossiers du CERI, mai 20II, p. I-I5.

KAHLOUN, H. (20I0). "What urban government for a sustainable urban planning? Conceptual analysis based on the case study of Tunisian cities and their local Agenda 21 », Environnement Urbain, vol. 4, p. c3l-c42. 


\section{EUE $\bullet$ SFAX $\bullet a-98$}

MEDCITES (201I). « Les Stratégies de Développement Urbain en Méditerranée: Contexte, Enjeux et Perspectives "), Conférence Stratégie de développement urbaine en Méditerranée, I33 p.

MEGDICHE, T. (20I0). " La « reconquête » du littoral de Sfax et ses enjeux ». In DLALA, H. (éd.), Mondialisation et changement urbain, Tunis, Centre de publication universitaire de Tunis, p. 139-160.

MEGDICHE, T. (20I2). « Taparura : genèse et enjeux d'un projet de réhabilitation du littoral ». In MESINI, B. (éd.), Aménagement durable des territoires méditerranéens, Aix-en-Provence, Publications de I'Université de Provence, p. 59-72.

MYLLYLÄ S. et K. KUVAJA (2005). «Societal premises for sustainable development in large southern cities», Global Environmental Change Part A, vol. 15, no 3, p. 224-237.

NAVEZ-BOUCHANINE F. (2007). «Le développement urbain durable: «best practice» ou leurre méthodologique ?», Espaces et sociétés [En ligne], vol. I3I, no 4, URL : http://dx.doi.org/10.3917/esp.131.0101. [consulté le 16 mai 2012]

ROBINSON, J. (20II). " The spaces of circulating knowledge: city strategies and global urban governmentality ॥. In MCCANN E. et K. WARD (éd.), Mobile Urbanism. Cities and Policymaking in the Global Age, Minneapolis, University of Minnesota Press, p. 1540.

RONCAYOLO, M. (1990). L'imaginaire de Marseille: port, ville, pôle, Marseille, Chambre de commerce et d'industrie de Marseille, $380 \mathrm{p}$.

SOUAMI, T. (20II). «Les Suds face au modèle nordeuropéen des quartiers durables: exploration d'expériences au Maghreb». In BARTHEL P.-A. et L. ZAKI (éd.), Expérimenter la « ville durable » au sud de la Méditerranée. Chercheurs et professionnels en dialogues, La Tour-d'Aigues, Editions de l'Aube, p. I33-I58.

VERDEIL, E. (20II). « Le développement urbain durable dans la Tunisie post-Ben Ali », Rumor [En ligne], 26 avril 20II, URL: http://rumor.hypotheses.org/I560, consulté le 17 septembre 2012 . 